\title{
DIGITAL CONTROLLERS PERFORMANCE ANALYSIS FOR A ROBOT ARM ${ }^{*}$
}

\author{
Abdullah Ahmed AL-DULAIMI ${ }^{1}$, Mohammed Majid \\ ABDULRAZZAQ ${ }^{2}$, Mustafa Mohammed ALHASSOW ${ }^{3}$, \\ Noor Qasim ALSAEDI ${ }^{4}$
}

\begin{abstract}
${ }^{1}$ Karabük University, Department of Electrical Electronics Engineering, Karabük, Turkey, abdalluhahmed1993@gmail.com; ORCID:0000-0001-8741-9450

${ }^{2}$ Karabük University, Department of computer Engineering, Karabük, Turkey moh.abdulrazzaq9@gmail.com; ORCID: 0000-0003-1850-9473

${ }^{3}$ Altinbas University, Department of Electrical and Computer Engineering, Istanbul, Turkey,mustafaalshakhe@gmail.com; ORCID:0000-0002-1757-7872

${ }^{4}$ Karabük University, Department of Electrical Electronics Engineering, Karabük, Turkey,noorqasimat@gmail.com; ORCID:0000-0002-6818-2459
\end{abstract}

\begin{abstract}
The design methodology and performance study of various forms of digital compensators for a robot arm joint control system with sensor feedback are presented in this article. Continuous time (s-plane or w-plane) and Discrete (z-plane) domain parameters are used in the design process. The frequency response characteristics design techniques were investigated, and five basic types of controllers were modelled and simulated using MATLAB: phase-lag, phase-lead, proportionalintegral (PI), proportional-derivative (PD), and proportional-integral derivative (PID). Many of the controllers have been set up to maintain a 40-degree phase margin. Both closed loop phase answers, as well as open loop bode plots, have been analyzed. This paper presents a comparison of the controllers based step response characteristics.
\end{abstract}

Keywords: Digital controllers, PID controllers, Robot arm, Robot arm controllers, Digital Controllers Performance.

\section{INTRODUCTION}

Controllers are needed to assess adjustments in system parameters and to meet performance requirements for steady-state precision, transient reaction, reliability, and disturbance prevention. Analog control systems are stable, with no intrinsic band width limitations or system changes. Due to the tolerances of practical machines, in analog controls, intricate logics are difficult to synthesize, while rendering complex interfaces

1* Received: 12.07.2021 - Accepted: 06.08.2021

DOI: 10.17932/EJEAS.2021.024/ejeas_v01i2003 
among multiple subsystems is very difficult, and are vulnerable to incorrect designs and limitations. Furthermore, extraneous noise sources can corrupt analog systems significantly. High-tech digital controls since no signal loss occurs in an along to digital (A/D) and digital to analog (D/A) conversions [1], systems are reliable. Furthermore, with a more sophisticated logic implementation, systems are more flexible and accurate. Filters do not encounter external noises, which makes them well-suited for adaptive filtering uses. Fast response and a digital memory interface are possible for digital systems [1]. A physical planet or system is accurately controlled through closed-loop or feedback operation where an output (system response) is adjusted as required by an error signal [2]. The discrepancy between the sponge as determined by sensor input and the target response generates the error signal. The error quantity is processed by a controller or compensator in order to satisfy those output requirements [3]. This paper describes five digital controller design methodologies for a robot control system in real-time. In these design methods, the compensating parameter is the phase margin specified in the plant bode diagram. The design method employs strategies of frequency response that allow for frequency cross-over phase margin (Pm). Phase-lag, PI and PID controllers (lag, lead) were drawn up in accordance with the principle of compensation and the methodologies as defined in [4]. This paper clarified the statistical and conceptual pricing articulated in the references. The primary and illustrative frames and approaches to digital control systems are mentioned in [6]. Digital control systems have been documented for training, theory, simulation and experimental approaches [7],[8]. A closed-loop model has been introduced in [1] and [4] for digital systems control and implementations in the Digital Drive Controller. Regarding to PID controller we citation the information from [5].

\section{METHODOLOGY}

A sampler, D/A block that is a zero-order hold ( $\mathrm{ZOH})$, a servomotor represented by an sdomain transfer mechanism, digital controller block, a power amplifier gain, gears represented by a gain value, and a feedback sensor block comprise the example robot control scheme outlined in this article. A s-domain transfer function presents the uncompensated plant. A/D conversion is started by the sampler and $\mathrm{D} / \mathrm{A}$ conversion is held at zero order. Controllers must offset the margin of the plant phase and the desired result shall be $40 \mathrm{deg}$. For each controller, steady-state error, percent overshoot, rise time, and settling time are calculated for output assessment. This paper section by section documents a literature review of digital compensation, an example uncompensated robot arm joint plant, discrete and continuous-time equations with design method, MATLAB simulation results of lag, lead, PI, PD and PID controls, and a comparative study among these five. In order to evaluate design requirements, the digital system was adopted, simulated and extended in MATLAB.

\section{LITERATURE REVIEW}

The compensation theory, plant configuration and the mathematical derivations of design approaches, loop parameters and open loop of the controllers mentioned in this paper fully follow the literature provided in [1]. The controller transfer function for first-order compensation can be written as 


$$
D(z)=\frac{K_{d}\left(z-z_{0}\right)}{z-z_{p}}
$$

Here, $z_{0}$ and $z_{p}$ represent the zero and pole positions, respectively. The controller's bilinear or trapezoidal transformation from the discrete $z$-plane to the continuous w-plane (warped s-plane) implies $D(w)=D(z), z=\frac{1+(T / 2) w}{1-(T / 2) w}$ and $\mathrm{D}(\mathrm{w})=\mathrm{a}_{0} \frac{1+\left(\mathrm{w} / \omega_{\mathrm{w} 0}\right)}{1+\left(\mathrm{w} / \omega_{\mathrm{wp}}\right)}$, Here $\omega_{\omega 0}$ and $\omega_{\omega p}$ denotes the zero and pole positions in the w-plane, and $\mathrm{a}_{0}$ denotes the compensator dc gain. The bilinear approximation states that:

$$
w=\frac{2}{T} \frac{z-1}{z+1}
$$

From the equations (1)-(4), in the z-plane the controller can be realized as

$$
D(z)=a_{0} \frac{\omega_{w p}\left(\omega_{w 0}+2 / T\right)}{\omega_{w 0}\left(\omega_{w p}+2 / T\right)} \frac{z-\left(\frac{2 / T-\omega_{w 0}}{2 / T+\omega_{w 0}}\right)}{z-\left(\frac{2 / T-\omega_{w p}}{2 / T+\omega_{w p}}\right)}
$$

The equation (1) yields to

$$
\begin{aligned}
K_{d} & =a_{0} \frac{\omega_{w p}\left(\omega_{w 0}+2 / T\right)}{\omega_{w 0}\left(\omega_{w p}+2 / T\right)} \\
z_{0} & =\frac{2 / T-\omega_{w 0}}{2 / T+\omega_{w 0}} \\
z_{p} & =\frac{2 / T-\omega_{w p}}{2 / T+\omega_{w p}}
\end{aligned}
$$

\section{BLOCK DIAGRAM EXPLANATION}

A closed-loop model for digital control systems and applications of digital controllers to speed drives has been shown in the above diagram. Thus, consists of a sampler, digital controller block, D/A block which is a zero-order hold $(\mathrm{ZOH})$, a power amplifier gain, a servomotor represented by a s-domain transfer function, gears represented by a gain value and a feedback sensor block. In the case of a closed-loop feedback system, the D(z) digital controller system is implemented. The controller uses algebraic algorithms such as filters and compensatory controls to correct or regulate the controlled system's behavior. The zero-order hold is a practical mathematical model of signal reconstruction using a digitalto-analog converter $(\mathrm{ZOH})$. This can be illustrated by you take a and convert it to a continuous-time signal, at a set time, it stores each sample value and does not allow changes. The amplitude or power of a signal input to output port can be increased by connecting it to an amplifier whose gain is set to a particular level [9]. 


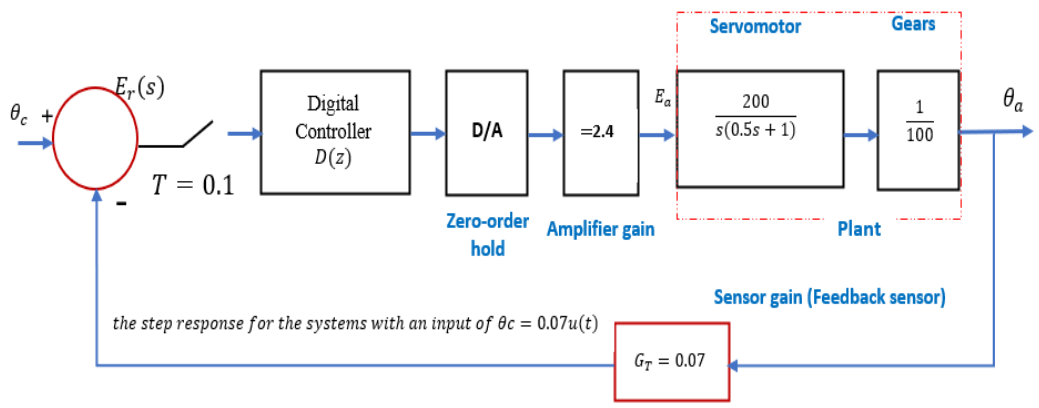

Figure 1. Robot arm joint control system block diagram

In order for a servomotor to transform the control signal from the controller into the rotational angular displacement or angular velocity of the motor output shaft, implies it has a servomotor. To power the arms of the robot, Servo motors are used. Gears are used to transfer motion. By finding the torque and speed of the output gear, you can find the torque and speed of the input gear. The uncompensated plant is presented by a s-domain transfer function. The sampler initiates A/D conversion and zero-order hold implements $\mathrm{D} / \mathrm{A}$ conversion. For performance evaluation, steady-state error, percent overshoot, rise time and settling time are measured for each controller. Here the given sensor feedback gain, $\mathrm{GT}=0.07$. The sensor input is $\theta_{a}$ In degrees and the output is in volts.

\section{PLANT}

The control system of the robot arm has been shown in Fig. 1. This system has shown the sampling time, $T=0.1 \mathrm{~s}$, the power amplifier increase, $\mathrm{K}=2.4$ and the sensor feedback gain, $H_{k}=0.07$. The system phase margin with, $D(z)=1$. The $\mathrm{ZOH}-\mathrm{TF}$ can be defined as

$$
G_{H O}(s)=\frac{1-e^{-s T}}{s}
$$

The plant TF in continuous time

$$
G_{p}(s)=\frac{9.6}{s^{2}+2 s}
$$

The sensor gain feedback TF is used in a continuous-time plant

$$
G_{c}(s)=G_{p}(s) \times H_{k}=\frac{0.672}{s^{2}+2 s}
$$

Where the TF is transfer function.

The sensor gain feedback TF that operates in discrete time is known as a discrete-time plant

$$
G_{d} \frac{0.00028289(s+3.39 e 04)}{(s+1.524)(s+0.4406)}
$$


Fig. 2. Introduces $D(z)=1$ system bode diagram, $P m$, for the uncompensated system is $79.6 \mathrm{deg}$ with a gain margin $\mathrm{Gm}=35.8 \mathrm{~dB}$.

\subsection{DESIGN OF PHASE LAG CONTROLLER}

Design a phase-lag controller with a dc gain of 10 that yields a system phase margin

$$
G_{h f}(d B)=20 \log \frac{a_{0} \omega_{w p}}{\omega_{w 0}}
$$

The controller in this paper is built for 39.7842 degrees. For this design, the Pm and the cross-over or Pm frequency have been chosen as $\omega_{w c}=1.1291 \mathrm{rads}^{-1}$.

$$
\omega_{w 0}=0.1 \omega_{w c}
$$

and

$$
\omega_{w p}=\frac{\omega_{w 0}}{a_{0}\left|G_{d}\left(j \omega_{w c}\right)\right|}
$$

The TF of the controller is

$$
D_{\text {lag }}(z)=\frac{0.01167(z-0.7105)}{(z-0.9919)}
$$

Figure 5 shows the phase-lag controller. The compensated plant $\mathrm{Pm}, \mathrm{Pm}=$ $39.7842 \mathrm{deg}$ at $0.0925 \mathrm{rad} / \mathrm{s}$, can be seen on the bode plot. $\mathrm{Gm}=$ $29.6 d B$ at $0.575 \mathrm{rad} / \mathrm{s}$. The gain and phase margin values are unknown in the marginalized bode plot of the controller, and hence these are determined to be infinite. The gain and phase margin values are unknown in the Bode plot of the controller, and hence these are found to be infinite where the Pm is the phase margin.

\subsection{DESIGN OF PHASE LEAD CONTROLLER}

The phase-lead controller, $\mathrm{a}_{0}=10$ and maximum phase shift, $\theta_{m}$ occurs at a frequency $\omega_{w m}=\sqrt{\omega_{w 0} \omega_{w p}}$. The controller in this paper is equipped for 39.8827 degrees for this design, the Pm and cross over or Pm frequency have been chosen as $2.2950 \mathrm{rad} / \mathrm{s}$. a phase-lead design controller with a dc gain of 10 that yields a system phase margin 40 deg.

$$
D\left(j \omega_{w c}\right) G_{d}\left(j \omega_{w c}\right)=1 \angle\left(180+\phi_{p m}\right)
$$

Here $\phi_{p m} \mathrm{pm}$ is the desired Pm and

$$
D(w)=a_{0} \frac{1+w /\left(a_{0} / a_{1}\right)}{1+w /\left(b_{1}\right)^{-1}}
$$

Where $\omega_{w 0}=\mathrm{a}_{0} / a_{1}$ and $\omega_{w p}=1 / b_{1}$. The angle can be described as.

$$
\theta_{r}=\angle D\left(j \omega_{w c}\right)=180+\phi_{p m}-\angle G_{d}\left(j \omega_{w c}\right)
$$


The controller design requires

$$
\left|D\left(j \omega_{w c}\right)\right|=\frac{1}{\left|G_{d}\left(j \omega_{w c}\right)\right|}
$$

from the equation (16)-(18), it can be evaluated that

$$
a_{1}=\frac{1-a_{0}\left|G_{d}\left(j \omega_{w c}\right)\right| \cos \theta_{r}}{\omega_{w c}\left|G_{d}\left(j \omega_{w c}\right)\right| \sin \theta_{r}}
$$

and

$$
b_{1}=\frac{\cos \theta_{r}-a_{0}\left|G_{d}\left(j \omega_{w c}\right)\right|}{\omega_{w c} \sin \theta_{r}}
$$

Because of the phase lead characteristic, $\theta_{r}>0$ and in the design procedure $\omega_{w c}$ has been constrained by the following requirements

$$
\begin{aligned}
& \angle G_{d}\left(j \omega_{w c}\right)<180+\phi_{p m} ;\left|D\left(j \omega_{w c}\right)\right|>a_{0} \\
& \left|G_{d}\left(j \omega_{w c}\right)\right|<\frac{1}{a_{0}} ; b_{1}>0 \\
& \cos \theta_{c}>a_{0}\left|G_{d}\left(j \omega_{w c}\right)\right|
\end{aligned}
$$

The transfer function of the controller is

$$
D(z)=\frac{10.424 *(z-0.9832)}{(z-0.9618)}
$$

From the bode plot, it can be observed that the compensated plant $\mathrm{Pm}, P_{m}=$ $39.8827 \mathrm{deg}$. At $2.29 \mathrm{rad} / \mathrm{s}$ and the gain margin, $G_{m}=16.2 \mathrm{~dB}$ at $6.57 \mathrm{rad} / \mathrm{s}$. From the bode plot of controller figure, in fact, it is evident that the gain and Pm values are undefined and thereby these are found to be infinite.

\subsection{DESIGN OF PI CONTROLLER}

PI controller means Proportional Integral controller it is composite of proportional and integral controller. They are in cascade with each other, as we see in fig.

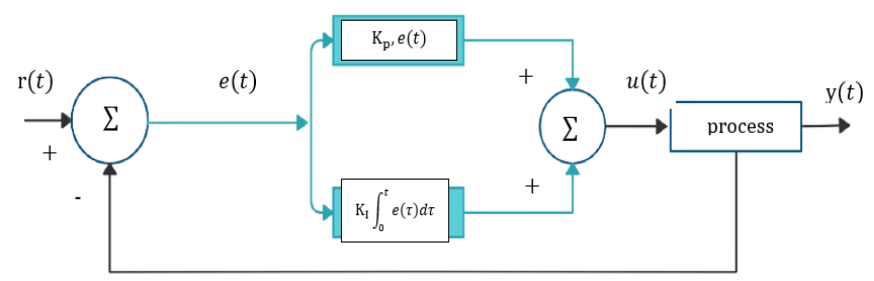

Figure 2. THE DESIGN OF PI CONTROLLER

The TF of the controller can be expressed as

$$
D(w)=K_{P}+\frac{K_{I}}{w}=K_{I} \frac{1+w / \omega_{w 0}}{w}
$$


Where $\omega_{w 0}=K_{I} / K_{P}$. However, the discrete TF of a PI controller can be expressed as

$$
D(z)=K_{P}+K_{I} \frac{T}{2} \frac{z+1}{z-1}
$$

PI controller design that yields a system phase margin with $40 \mathrm{deg}$

$$
D\left(j \omega_{w c}\right) G_{d}\left(j \omega_{w c}\right)=1 \angle\left(-180+\phi_{p m}\right)
$$

Let $\mathrm{A}=\mid G_{d}\left(j \omega_{w c} \mid\right.$, The $K p$ proportional gain and $K_{I}$ integral gain can be expressed as

$$
\begin{aligned}
& K p=\frac{\cos \theta_{r}}{\mid G_{d}\left(j \omega_{\omega_{c}} \mid\right.} \\
& K_{I}=\frac{\omega_{w c} \sin \theta_{r}}{\left|G_{d}\left(j \omega_{\omega c}\right)\right|}
\end{aligned}
$$

The TF of the controller is

$$
D(z)=\frac{1.4575(z-0.9648)}{(z-1)}
$$

From the bode plot, this is worth noting as that the compensated plant $\mathrm{Pm}, P_{m}=$ $40.0182 \mathrm{deg}$. At $0.553 \mathrm{rad} / \mathrm{s}$ and the gain margin, $G_{m}=30.9 \mathrm{~dB}$ at $5.61 \mathrm{rad} / \mathrm{s}$. From the bode plot of controller figure, it can be observed that the gain and Pm values are undefined and thereby these are found to be infinite.

\subsection{DESIGN OF PD CONTROLLER}

PD controller means proportional derivative Controller so it has both, the proportional controller and derivative controller in cascade, so we have to add both, as we see in fig.

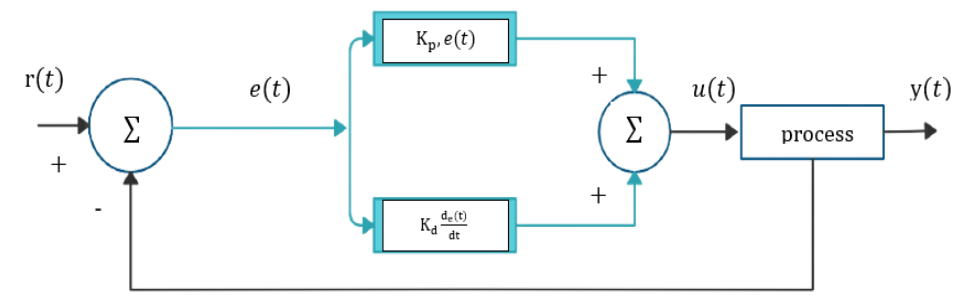

Figure 3. THE DESIGN OF PD CONTROLLER

Let $w_{1}$ the Gain crossover frequency of the system with cascade PD controller, $A_{1}$ the $\left|G_{1}(j \omega)\right|$ at $\omega_{1}$. The PI controller transfer function is

$$
D(\omega)=k_{p}+k_{D} w
$$


Moreover, we can change the transfer function from w-plane to $z$-transform using the bilinear transformation. In Bilinear transformation, $\omega$ is replaced by $\frac{2}{T}\left(\frac{z-1}{z+1}\right)$, where $T=$ sampling time.

the discrete TF of a PI controller is

$$
D(z)=k p+k_{D_{T}^{2}}^{2}\left(\frac{z-1}{z+1}\right)
$$

controller design that yields a system phase margin with $40 \mathrm{deg}$

$$
-k_{d} w_{1}^{2}+k_{i}+j k p w_{1}=\frac{\omega_{1}}{A_{1}} \sin \theta+j \frac{\omega_{1}}{A_{1}} \cos \theta
$$

The derivative gain and proportional gain can be expressed as

$$
\begin{aligned}
k_{d} & =\frac{\sin \theta}{\omega, A_{1}} \\
\therefore k_{\mathrm{d}} & =\frac{\sin \theta}{\omega_{1}\left|G\left(j \omega_{1}\right)\right|} \\
k_{p} & =\frac{\cos \theta}{A_{1}} \\
\therefore k_{p} & =\frac{\cos \theta}{\left|G\left(j \omega_{1}\right)\right|}
\end{aligned}
$$

\subsection{DESIGN OF PID CONTROLLER}

PID controller proportional Integral Derivative controller Consists of proportional, integral, and Derivative controller all connected in the cascade form, as we see in fig.

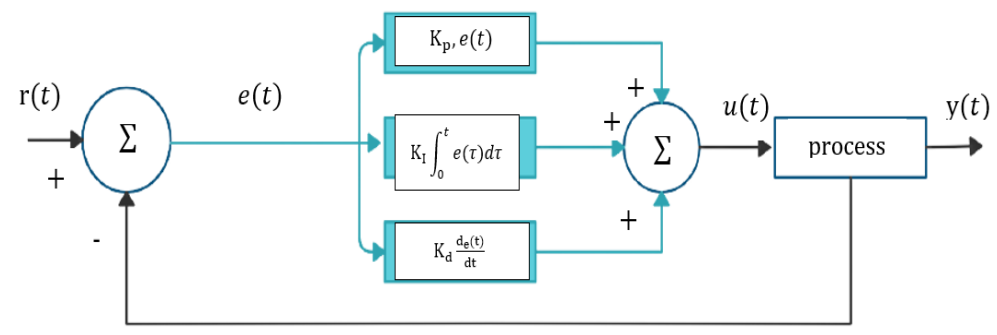

Figure 4. THE DESIGN OF PID CONTROLLER

$$
D(w)=K_{P}+\frac{K_{I}}{w}+K_{D} w
$$

Moreover, we can change the transfer function from w-plane to $z$ - transform by using the bilinear transformation. In Bilinear transformation $\omega$ is replaced by $\frac{2}{T}\left(\frac{z-1}{z+1}\right)$, where $T=$ sampling time. 
The PID controller's discrete TF can be expressed as

$$
D(z)=K_{P}+K_{I} \frac{T}{2} \frac{z+1}{z-1}+K_{D} \frac{z-1}{T z}
$$

controller design that yields a system phase margin with $40 \mathrm{deg}$

$$
\left[K_{P}+\frac{K_{D} \omega_{w c}^{2}(2 / T)}{(2 / T)^{2}+\omega_{w c}^{2}}\right]+j\left[\frac{K_{D} \omega_{w c}(2 / T)^{2}}{(2 / T)^{2}+\omega_{w c}^{2}}-\frac{K_{I}}{\omega_{w c}}\right]=K_{R}+j K_{C}
$$

The $K_{P}$ proportional gain, $K_{D}$ derivative gain and $K_{I}$ integral gain can be expressed as the controller transfer function, which can be expressed as

$$
\begin{aligned}
& \therefore K_{P}+\frac{K_{D} \omega_{w c}^{2}(2 / T)}{(2 / T)^{2}+\omega_{w c}^{2}}=\frac{\cos \theta_{r}}{\left|G_{d}\left(j \omega_{w c}\right)\right|} \\
& \therefore \frac{K_{D} \omega_{w c}(2 / T)^{2}}{(2 / T)^{2}+\omega_{w c}^{2}}-\frac{K_{I}}{\omega_{w c}}=\frac{\sin \theta_{r}}{\left|G_{d}\left(j \omega_{w c}\right)\right|}
\end{aligned}
$$

The gain margin is $=20 \mathrm{~dB}$ at $6.99 \mathrm{rad} / \mathrm{s}$, and the $\mathrm{Pm}$ is $=38.1$ degrees by the PID controller at $1.85 \mathrm{rad} / \mathrm{s}$. The gain and phase margin values are unknown in the marginalized bode plot of the controller, and hence these are determined to be infinite. 


\subsection{CONTROLLERS' BODE PLOT}

The curves and the table below are for all the controllers discussed in the previous sections.

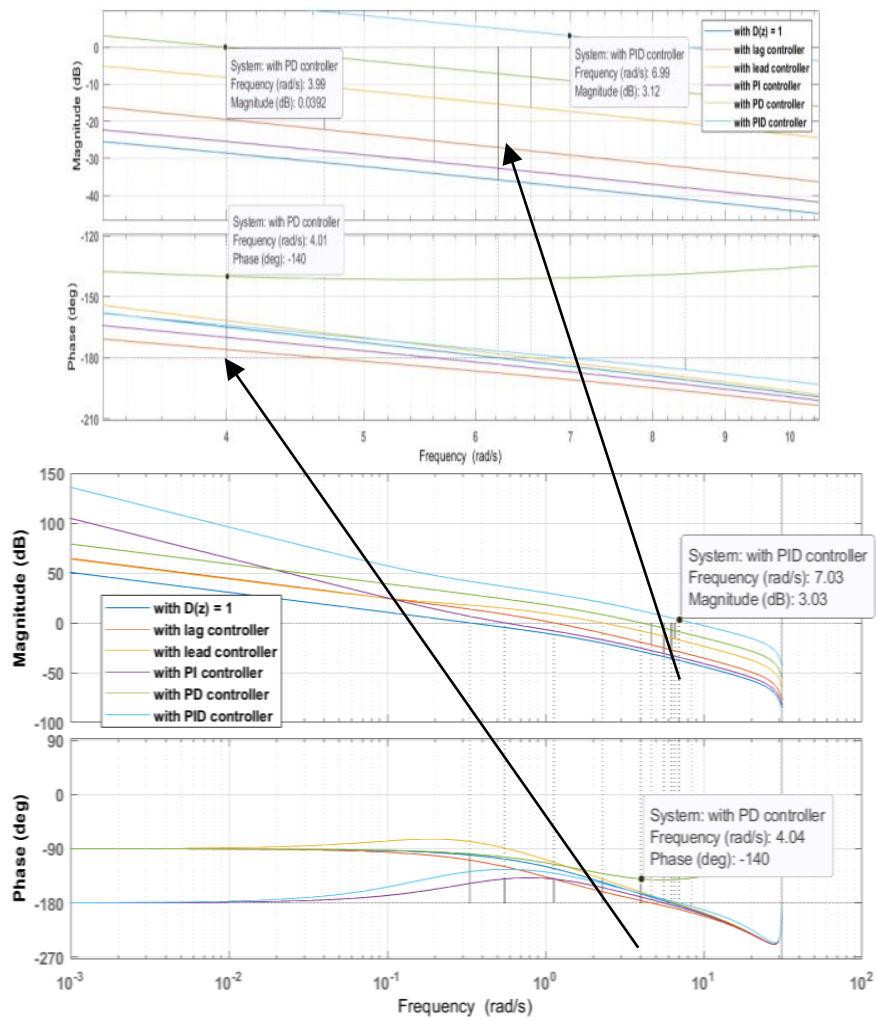

Figure 5. Controllers' Bode plot of the open loop

Table 1. Controllers' Bode plot characteristics

\begin{tabular}{|c|c|c|c|c|c|c|}
\hline Characteristics & $\begin{array}{c}\text { Pm with } \mathrm{D}(\mathrm{z}) \\
=1\end{array}$ & Lag & Lead & PI & PD & PID \\
\hline Gain Margin & $\begin{array}{c}61.5755 \\
1.7929 \mathrm{e}+04 \\
\end{array}$ & $\begin{array}{c}12.8749 \\
6.7538 \mathrm{e}+0 \\
\end{array}$ & $\begin{array}{l}6.49351 .7 \\
014 \mathrm{e}+03 \\
\end{array}$ & $\begin{array}{l}09.97021 . \\
8455 \mathrm{e}+03\end{array}$ & Inf & $\begin{array}{c}09.9702 \\
1.8455 \mathrm{e}+03 \\
\end{array}$ \\
\hline $\begin{array}{c}\text { GM } \\
\text { Frequency }\end{array}$ & $\begin{array}{r}6.2240 \\
31.4159 \\
\end{array}$ & $\begin{array}{r}4.6917 \\
31.4159 \\
\end{array}$ & $\begin{array}{l}6.5659 \\
31.4159 \\
\end{array}$ & $\begin{array}{l}06.9890 \\
31.4159 \\
\end{array}$ & Inf & $\begin{array}{l}06.9890 \\
31.4159 \\
\end{array}$ \\
\hline Phase Margin & 79.6399 & 39.7842 & 39.8827 & 38.1362 & 40.0 & 38.1362 \\
\hline $\begin{array}{c}\text { PM } \\
\text { Frequency }\end{array}$ & 0.3315 & 1.1291 & 2.2950 & 1.8486 & $\begin{array}{c}4.003 \\
5\end{array}$ & 1.8486 \\
\hline Delay Margin & 41.9333 & 6.1499 & 3.0331 & 3.6006 & $\begin{array}{c}1.743 \\
8\end{array}$ & 3.6006 \\
\hline $\begin{array}{c}\text { DM } \\
\text { Frequency }\end{array}$ & 0.3315 & 1.1291 & 2.2950 & 1.8486 & $\begin{array}{c}4.003 \\
5 \\
\end{array}$ & 1.8486 \\
\hline Stable & 1 & 1 & 1 & 1 & 1 & 1 \\
\hline
\end{tabular}




\section{STEP RESPONSE CHARACTERISTICS}

Design problem explained in this paper has assumed an input of $\theta_{-} \mathrm{c}=0: 07 \mathrm{u}(\mathrm{t})$. The controller scaled step response of the closed-loop system for the design is presented in Figure 6. For the step response overshoot, $\left.\vdash \xi \downarrow \Rightarrow M \_p \%(\% O S)\right) \uparrow$. From figure 6 and table 2 , the PID controller is the best because it has less steady state error than other controllers. For PID $\square$ steady-state error $\propto 1 / \mathrm{k} \_p$, OS $\propto 1 / \mathrm{k} \_\mathrm{d}$, Rise Time $\propto 1 / \mathrm{k} \_\mathrm{p}, \propto 1 / \mathrm{k} \_\mathrm{i}$, Settling Time $\propto 1 / \mathrm{k} \mathrm{d}$. Kp, Kd, and $\mathrm{Ki}$ can be described as the proportional, derivative, and integral parameters. The closed-loop controls system is affected by all three of these parameters. In addition to those factors, the slow rising, slow settling, and long. Overshoot, as well as the steady state error, are also affected. A lag compensator shifts the Bode magnitude plot down at mid and high frequencies with its attenuation property. Highlights for specification on steady-state error, the low frequency gain is changed. The proportional-integral controller is equivalent to a control system that produces an output, this calls attention to which is the result of adding outputs from the proportional and integral controllers. PID is used in systems where proportional, integral, and derivative controllers are used to compute an output. Implies It's also there to reduce steady state error and improve stability. Reveals that when used in conjunction with a proportional and a derivative controller, the proportional derivative controller generates an output, which is the product of the proportional and derivative controllers. If PD is being used, noise may be suppressed in the higher frequencies.

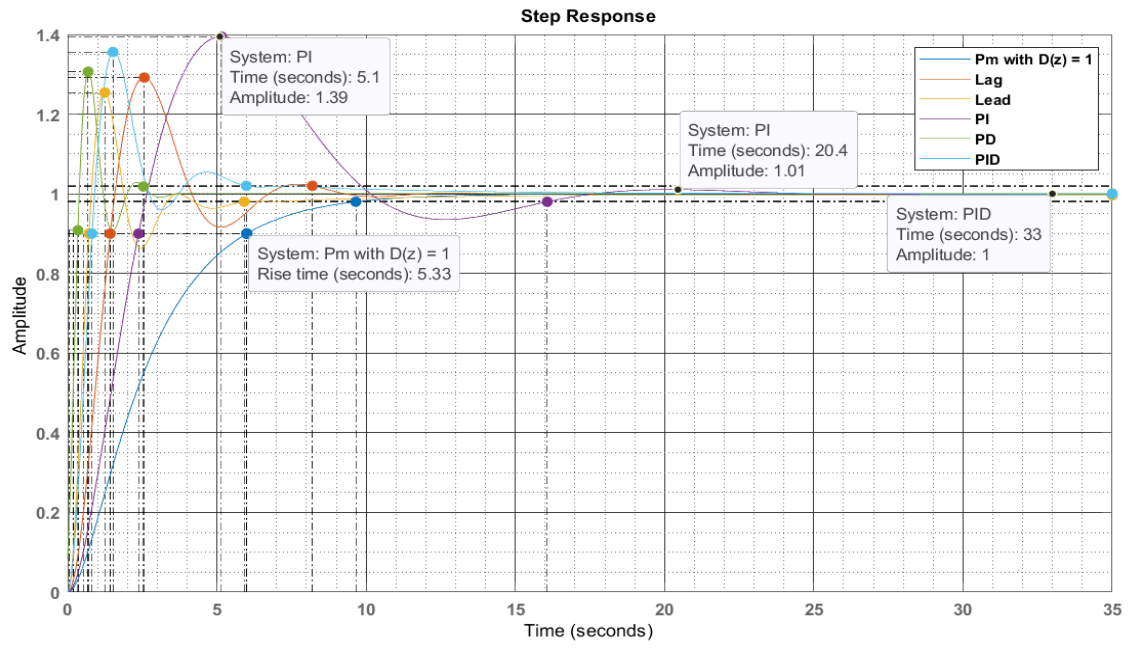

Figure 6. Controllers' step response of the closed loop 
Table 2. Controllers' step response characteristics

\begin{tabular}{|l|c|c|c|c|c|c|}
\hline Characteristics & $\begin{array}{l}\text { Pm with } \\
\text { D(z)= }\end{array}$ & Lag & Lead & PI & PD & PID \\
\hline Rise Time: & 5.3278 & 1.0700 & 0.5359 & 1.8526 & 0.2940 & 0.6182 \\
\hline Settling Time & 9.6546 & 8.1969 & 5.9127 & 16.0705 & 2.5379 & 5.9852 \\
\hline Settling Min & 0.9020 & 0.9004 & 0.8641 & 0.9053 & 0.9068 & 0.9321 \\
\hline Settling Max & 0.9986 & 1.2919 & 1.2543 & 1.3949 & 1.3064 & 1.3560 \\
\hline Overshoot & 0 & 29.1881 & 25.4273 & 39.4865 & 30.6421 & 35.6044 \\
\hline Undershoot & 0 & 0 & 0 & 0 & 0 & 0 \\
\hline Peak & 0.9986 & 1.2919 & 1.2543 & 1.3949 & 1.3064 & 1.3560 \\
\hline Peak Time & 15.6573 & 2.5692 & 1.2423 & 5.1509 & 0.6846 & 1.5198 \\
\hline
\end{tabular}

\section{CONCLUSION}

This paper examines the performance and design assessment of five simple digital controllers, including lag, lead, PI, PD, and PID controllers, which are used for a physical robot arm joint plant. C-O frequency is a crucial design specification to compensate the plant. Implies design a system controller with a dc gain of 10 that yields a system phase. The design methodologies have been investigated in both discrete $z$-domain time approaches and warped s-domain or w-plane time frames. The controllers have been simulated on MATLAB and bode plots with open loop and closed loop step response curves have been analyzed for comparative margin Pm 40 deg. Premises. The suggests tells us such design is crucial as its specifications are applicable in different practical control systems.

\section{REFERENCES}

[1] Chowdhury, Dhiman. "Design and Performance Analysis of Digital Controllers in Discrete and Continuous Time Domains for a Robot Control System." Global Journal of Research In Engineering (2018).

[2] Unglaub, Ricardo AG, and D. Chit-Sang Tsang. "Phase tracking by fuzzy control loop." 1999 IEEE Aerospace Conference. Proceedings (Cat. No. 99TH8403). Vol. 5. IEEE, 1999.

[3] Mastinu, Gianpiero, and Manfred Plöchl, eds. Road and off-road vehicle system dynamics handbook. CRC press, 2014.

[4] Chowdhury, Dhiman, and Mrinmoy Sarkar. "Digital Controllers in Discrete and Continuous Time Domains for a Robot Arm Manipulator." arXiv preprint arXiv:1912.09020 (2019).

[5] Alassar, Ahmed Z., Iyad M. Abuhadrous, and Hatem A. Elaydi. "Comparison between FLC and PID Controller for 5DOF robot arm." 2010 2nd International Conference on Advanced Computer Control. Vol. 5. IEEE, 2010. 
[6] Phillips, Charles L., and H. Troy Nagel. Digital control system analysis and design. Prentice-Hall, Inc., 1989.

[7] Misir, Dave, Heidar A. Malki, and Guanrong Chen. "Design and analysis of a fuzzy proportional-integral-derivative controller." Fuzzy sets and systems 79.3 (1996): 297-314.

[8] Klee, Harold, and Joe Dumas. "Theory, simulation, experimentation: an integrated approach to teaching digital control systems." IEEE transactions on education 37.1 (1994): 57-62.

[9] Liu, Hui. Robot Systems for Rail Transit Applications. Elsevier, 2020.

[10] Boukas EK., AL-Sunni F.M. (2011) Design Based on Transfer Function. In: Mechatronic Systems. Springer, Berlin, Heidelberg. https://doi.org/10.1007/978-3-642-22324-2_5. 